\title{
Bandwidth Enhancement in an Integratable SiGe Phototransistor by Removal of Excess Carriers
}

\author{
Z. Pei, J.-W. Shi, Y.-M. Hsu, F. Yuan, C. S. Liang, S. C. Lu, W. Y. Hsieh, M.-J. Tsai, and \\ C. W. Liu, Senior Member, IEEE
}

\begin{abstract}
In this letter, we create a path to remove excess carriers in the base region of a SiGe phototransistor (HPT) by introducing the trap centers. The behavior of the trap centers in the SiGe heterojunction bipolar transistor (HBT) is a form of nonideal (nkT) base current. The responsivity of the device is $\sim 0.43 \mathrm{~A} / \mathrm{W}$ with fully SiGe HBT-compatible device structure to facilitate the integration of the following amplification circuitry. The full-width at half-maximum of the pulse is $\sim 90 \mathrm{ps}$ and the tail of the optical pulse response is largely reduced with the nkT current. By reducing the tail, bandwidth is increased from 1.5 to $3 \mathrm{GHz}$. This proposes SiGe HPT is applicable for optoelectronic technology.
\end{abstract}

Index Terms-Bandwidth, nonideal (nkT) base current, SiGe.

\section{INTRODUCTION}

$\mathbf{S}$ Ge phototransistors (HPT) with SiGe-Si multiple quantum well (MQW) absorption layers have been demonstrated with high responsivity, high bandwidth, infrared (1.31 and $1.55 \mu \mathrm{m})$ detection ability, and can be used for high-speed optical communication applications [1]-[3]. The optical impulse response shows the pulse width is as low as $200 \mathrm{ps}$, which could have gigahertz operation ability. However, the 3-dB bandwidth is limited to $\sim 500 \mathrm{MHz}$ after Fourier transform due to the serious signal tail while the optical input pulse shutoff. Moreover, the photodetectors with multigigahertz bandwidth is highly demanded for the future high-speed optical communication. Methods should be developed to eliminate the long tail in a HPT and then increasing the bandwidth to multigigahertz. In addition, to facilitate the integration with the following amplification circuitry, the SiGe-Si MQW absorption layers can be removed for the 850-nm wavelength detection.

The tail in a Si photodetector is generally described by the absorption of Si substrate that exhibit a long diffuse time. The contribution form substrate absorption is verified experimentally by measuring the pulse signal of a Si photodetector fabricating on a silicon-on-insulator substrate that could blocking the substrate signal [4]. However, in an HPT, the photocurrent from the light

Manuscript received February 4, 2004; revised February 27, 2004. The review of this letter was arranged by Editor P. Yu.

Z. Pei, Y.-M. Hsu, C. S. Liang, S. C. Lu, W. Y. Hsieh, and M.-J. Tsai are with the Electronics Research and Service Organization (ERSO), ITRI, Hsinchu, Taiwan, R.O.C.

J.-W. Shi was with the Electronics Research and Service Organization (ERSO), ITRI, Hsinchu, Taiwan, R.O.C. He is now with the Department of Electrical Engineering, National Central University, Taoyuan 320, Taiwan, R.O.C.

F. Yuan and C. W. Liu are with the Electronics Research and Service Organization (ERSO), ITRI, Hsinchu, Taiwan, R.O.C. and also with Department of Electrical Engineering, National Taiwan University, Taipei, Taiwan, R.O.C. Digital Object Identifier 10.1109/LED.2004.826975 absorption in the base-collector depletion region is amplified by 100 times due to the "HPT" operation. As compared to this photocurrent, the absorption from the substrate should be a minor term that should not responsible for the serious tail in the pulse measurement. Instead, the photogenerated holes accumulated in the base-emitter could possible be the origin of the tail [5]. The accumulated holes in the base need a recovery time back to equilibrium state. At the meantime, the carriers still affect the base-emitter junction that could induce the electrons flow and cause the tail in the pulse measurement. In order to improve the speed performance of HPTs, dc bias voltage or current across base-emitter junction is necessary [5]. However, these traditional approaches will result in huge dark current, large power consumption, and reduction in operation gain [5]. In our demonstrated SiGe HPTs, by using the nonideal base current (nkT current) to remove the excess carriers in the base-emitter junction, the speed performance can be improved significantly without increasing dark current seriously. A fully integratable 3-GHz bandwidth SiGe HPT is demonstrated, the speed performance can be improved significantly without increasing dark current seriously.

\section{DEVICE DESIGN}

The path for the excess carriers removing after the optical pulse shutoff can be provided by the means of the nkT current at low currents in the $\mathrm{Si}$ or SiGe bipolar transistor. The nkT current is either the generation/recombination current in the trap centers of the base-emitter junction or the tunneling current due to the high doping at the base-emitter junction [6]. The trap centers for the recombination usually locate at $\mathrm{Si}-\mathrm{SiO}_{2}$ interface. The $\mathrm{Si}_{-} \mathrm{SiO}_{2}$ interfaces in SiGe HBT are either between the extrinsic-base and poly-silicon emitter (the spacer oxide) or at the isolation edge (local isolation or shallow trench isolation) [7]. The trap centers are in generally the oxidation-induced defects, or the bond breaking by the hot carriers. Thus, trap centers either exist in the fresh HBT or can be generated by base-emitter reverse-bais voltage stress or forward-base current stress [6], [7]. The high energy carriers could inject into the oxide and creating traps at oxide/Si interface by breaking weak bonds. The base leakage current was found to increase after the stress [8]. Two SiGe HPTs were used in this experiment, one (Device B) has very clear nkT current, another (device A) does not. The nkT current is selected from the "fresh" HBT to omit the un-controllable stress during the measurement. The fabrication process of SiGe HPT is described in detail elsewhere [3], [9]. The Gummel 


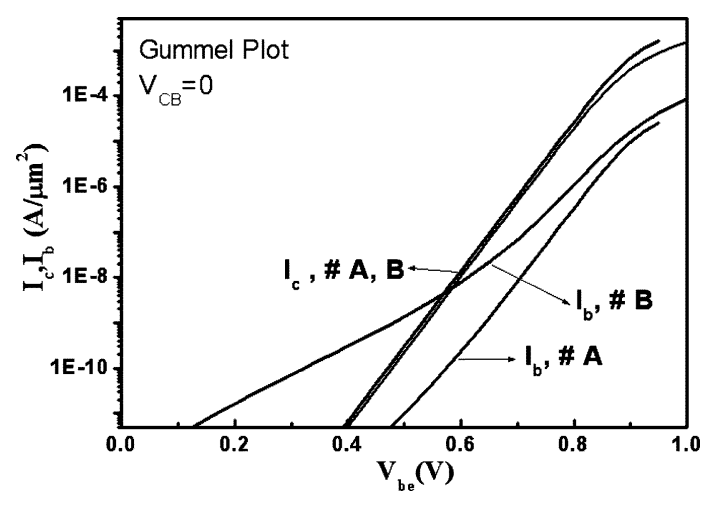

Fig. 1. Gummel plot of the SiGe HPT.

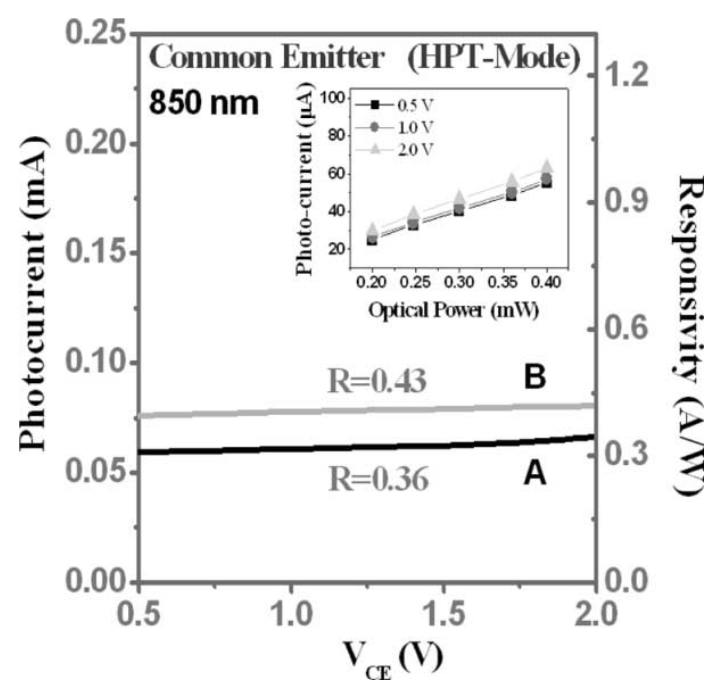

Fig. 2. Photocurrent and responsivity of the MQW HPT at 850-nm light illumination. Inset shows the photocurrent to input optical power relationship.

plot of the HPT is shown in Fig. 1. These two devices have almost the same collector current to focus on the effect of the base current. Device A has near-ideal bipolar performance, while device $\mathrm{B}$ has clear nkT current. The current gain for both devices is $\sim 100$.

\section{RESULTS AND DISCUSSION}

The photocurrent is measured by a continuous laser with center wavelength at $850 \mathrm{~nm}$. The output power is $180 \mu \mathrm{W}$. The laser is coupled to the device by a fiber probe. The bias and photocurrent were supplied and collected by a HP 4156 parameter analyzer. The photocurrents of device $\mathrm{A}$ and $\mathrm{B}$ are at range of $60 \sim 80 \mu \mathrm{A}$ for the entire bias range. The responsivity at $2 \mathrm{~V}$ for device $\mathrm{A}$ and $\mathrm{B}$ are 0.36 and $0.43 \mathrm{~A} / \mathrm{W}$, respectively. Inset of the Fig. 2 shows the photocurrent versus optical power $(200 \sim 400 \mu \mathrm{W})$ at $V_{\mathrm{CE}}$ bias of $0.5,1$, and $2 \mathrm{~V}$ for a SiGe HPT. The linear and bias voltage independent behavior indicates that the HPT is suitable for optical communication. The responsivity is smaller than the previous result, $1.2 \mathrm{~A} / \mathrm{W}$ [3], might be due to the remove of the SiGe MQWs. However, this value is useful for the optical communication applications. The dark current of device B as low as $5 \mathrm{pA} / \mu \mathrm{m}^{2}$ is obtained. Regarding the nkT base current, the back flow holes generated from base-collector impact ionization process would not induce

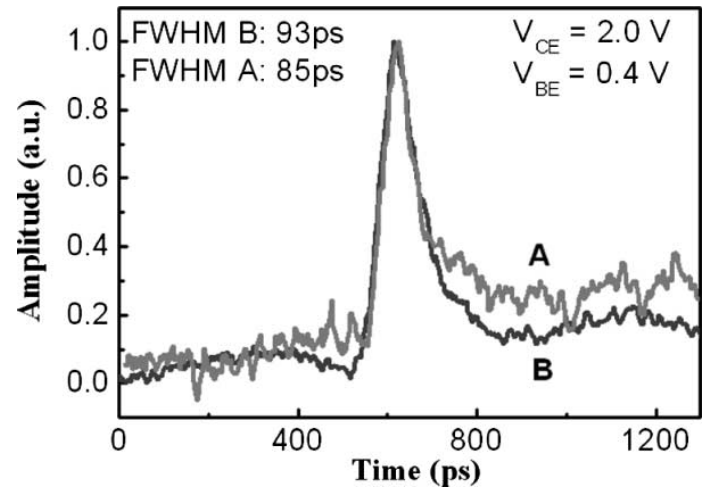

Fig. 3. Pulse response of SiGe HPT at $827-\mathrm{nm}$ and 50 -ps pulse laser illumination with the applied voltages of $2 \mathrm{~V}$.

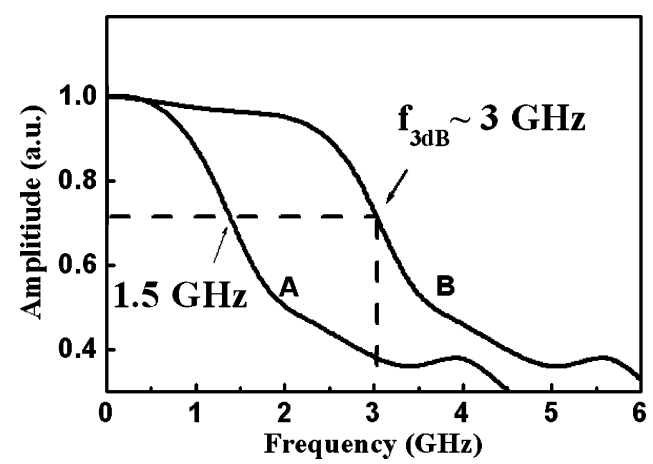

Fig. 4. Frequency response of the SiGe HPT after Fourier transform. The bandwidth is $\sim 3 \mathrm{GHz}$ for device $\mathrm{B}$.

extensive electron into the collector at the base open condition, due to the negligible current gain [6]. Therefore, a device with nkT base current could have lower dark current. The optical pulse response is performed similar to the DC measurement, except the laser is replaced by a HAMAMTSU pulse laser centered at $827 \mathrm{~nm}$. The pulse width is around $50 \mathrm{ps}$. The pulse response is recorded by a Tektronix digital sampling scope. Fig. 3 shows the pulse response of a SiGe HPT at $V_{\mathrm{CE}}=2 \mathrm{~V}$ and $V_{\mathrm{BE}}=0.4 \mathrm{~V}$. The rise time for sample $\mathrm{A}$ and $\mathrm{B}$ is $\sim 50 \mathrm{ps}$ and the full-width at half-maximum of the pulse response is $\sim 85 \mathrm{ps}$ for device $\mathrm{A}$ and $\sim 93 \mathrm{ps}$ for device $\mathrm{B}$. The behavior at fall time for two devices is quite different. The device A has more serious tail. The tail was largely reduced in device B. Fig. 4 shows the frequency response of device $A$ and $B$ after Fourier transform. The 3-dB bandwidths for device A and B are 1.5 and $3 \mathrm{GHz}$, respectively. The remove of tail cause the dramatic bandwidth increase from 1.5 to $3 \mathrm{GHz}$. The reduction of the tail at pulse measurement also indicates that the tail has come from the excess carriers, not the substrate absorption. The assumption of creative a path to remove the excess carriers after optical pulse takes effect, since the different between device $\mathrm{B}$ and $\mathrm{A}$ is, one has nkT current and the other one has not. Furthermore, the cut-off frequency $\left(f_{T}\right)$ and unit power gain frequency $\left(f_{\text {MAX }}\right)$ are appeared in high current range for SiGe HBT that the low voltage leakage current would not affect these values. Yang et al. [10] reported the $f_{T}$ has only slightly degraded after the stress. Therefore, the performance of following amplify circuit would not affect by the introduction of nkT current at SiGe HPT. 


\section{SUMMARY}

In summary, we report a high bandwidth of SiGe HPT by introducing the recombination path for excess carriers and remove the SiGe-Si MQWs. The path for the excess carriers to remove is the traps centers at $\mathrm{Si}_{-} \mathrm{SiO}_{2}$ interface and behave as the nkT current in the Gummel plot. The tail in the pulse response is reduced and the bandwidth is largely increased from 1.5 to $3 \mathrm{GHz}$ by introducing that path. The $3 \mathrm{GHz}$ bandwidth is fully suitable for $2.5 \mathrm{~Gb} / \mathrm{s}$ optical communication applications and the integrated optoelectronic circuit can be realized in this SiGe HPT structure.

\section{ACKNOWLEDGMENT}

The authors would like to thank Prof. Y. J. Chan of National Central University on pulse measurement for his support.

\section{REFERENCES}

[1] Z. Pei, C. S. Liang, L. S. Lai, Y. T. Tseng, Y. M. Hsu, P. S. Chen, S. C. Lu, C. M. Liu, M.-J. Tsai, and C. W. Liu, "High efficient 850-nm and 1310-nm multiple quantum well SiGe-Si heterojunction phototransistors with 1.25-plus GHz bandwidth $(850 \mathrm{~nm})$," in IEDM Tech. Dig., 2002, pp. 297-300
[2] Z. Pei, L. S. Lai, H. P. Hwang, Y. T. Tseng, C. S. Liang, and M.-J. Tsai, " $\mathrm{Si}_{1-x} \mathrm{Ge}_{x}-\mathrm{Si}$ multi-quantum well phototransistor for near-infrared operation," Phys. E, vol. 16, no. 3-4, pp. 554-557, 2003.

[3] Z. Pei, C. S. Liang, L. S. Lai, Y. T. Tseng, Y. M. Hsu, P. S. Chen, S. C. Lu, M.-J. Tsai, and C. W. Liu, "A high-performance SiGe-Si multiple-quantum-well heterojunction phototransistor," IEEE Electron Device Lett., vol. 24, pp. 643-645, Oct. 2003.

[4] M.Min Yang, J. Schaub, D. Rogers, M. Ritter, K. Rim, J. Welse, and B.Byeongju Park, "High-speed silicon lateral trench detector on SOI substrate," in IEDM Tech. Dig., 2001, pp. 547-550.

[5] M. Y. Frankel, T. F. Carruthers, and C. S. Kyono, "Analysis of ultrafast photocarrier transport in AlInAs-GaInAs heterojunction bipolar transistors," IEEE J. Quantum Electron., vol. 31, pp. 278-285, Feb. 1995.

[6] Y. Tuar and T. H. Ning, Fundamentals of Modern VLSI Devices. Cambridge, U.K.: Cambridge Univ. Press, 1998, pp. 323-327.

[7] R. A. Wachnik, T. J. Bucelot, and G. P. Li, "Degradation of bipolar transistors under high current stress at $300 \mathrm{~K}$," J. Appl. Phys., vol. 63, p. 4734,1988

[8] G. Zhang, J. D. Cressler, G. Niu, and A. Joseph, "A new 'mixed-mode' reliability degradation mechanism in advanced $\mathrm{Si}$ and SiGe bipolar transistors," IEEE Trans. Electron Devices, vol. 49, pp. 2151-2156, Dec. 2002.

[9] L. S. Lai, Y. T. Tseng, L. S. Lee, Y. S. Jean, Y. M. Hsu, H. P. Hwang, S. C. Lu, and M.-J. Tsai, "The high quality low temperature oxidation technology in a quasi self-aligned SiGe HBT," in Proc. Int. Symp. Electron Devices for Microwave and Optoelectronic Applications, 2001, pp. 77-82.

[10] Z. Yang, F. Guarin, E. Hostetter, and G. Freeman, "Avalanche current induced hot carrier degradation in $200 \mathrm{GHz}$ SiGe heterojunction bipolar transistors," in Proc. IEEE Int. Reliability Physics Symp., 2003, pp. 339-343. 